\title{
New Procedure for Assigning Drivers to Work Schedules at a Container Terminal
}

\author{
Khaled Mili ${ }^{1}$, Ilhem Elghoul ${ }^{2}$ \\ ${ }^{1}$ Department of Economics and Quantitative Methods and Computer, Institute of Companies Administration of Gafsa, Gafsa, Tunisia \\ ${ }^{2}$ Department of Computer Science, Mediterranean Institute of Nabeul, Nabeul, Tunisia
}

Email address:

Khaled.mili.1@ualaval.ca (K. Mili), ilhem.el.ghoul@gmail.com (I. Elghoul)

\section{To cite this article:}

Khaled Mili, Ilhem Elghoul. New Procedure for Assigning Drivers to Work Schedules at a Container Terminal. Journal of Investment and Management. Vol. 4, No. 1, 2015, pp. 1-8. doi: 10.11648/j.jim.20150401.11

\begin{abstract}
One of the success factors of a terminal is related to the time in port for the retrieval and transport of containers. Straddle carriers (SCs) are the pivotal axis around which the terminal transportation system evolves and the success or failure of that process is an indicator of the reliability of the container terminal. Over the last years, the deficiency of efficient control and coordination mechanisms in practice produced a relaxation of transportation principles. The valorization of the academic environment represents nowadays one of the most important research challenges. In this paper, we present a collaborative filtering recommender system able to manage the work schedule's assignment to straddle carrier's drivers in a container terminal and provide preliminary results on customer's satisfaction.
\end{abstract}

Keywords: Recommendation System, Collaborative Filtering, Straddle Carrier's Assignment

\section{Introduction}

The RADES container terminal in Tunisia, which is the leading Tunisian port for the movement of containers (Africa infrastructure Country Diagnostic 2009) well equipped with necessary modern facilities and academic experience, has opted to provide a Straddle carrier's assignment recommendation system in order to reduce the malpractices that threaten the integrity of the transportation process and enter a new era in which growing number of e-transparency systems will be employed.

The goal of this work is to explore all aspects of the transportation problem and employ an intelligent system that can be more accurate and provide better recommendations to the straddle carrier's drivers. A work schedule for a straddle carrier driver is provided in the form of a sequence of container groups and a number of containers. The adopted strategy is based on a collaborative filtering mechanism by analyzing user's behaviors.

The remainder of this paper is arranged as follows: Section 2 gives the necessary background on collaborative filtering recommendation systems. Section 3 introduces the transportation management problem and the optimization model to solve it. In section 4 , we give the details of the proposed implementation. Concluding remarks and future works are given in Section 5.

\section{Background}

Recommendation systems help users find and select items of interest based on their explicit and implicit preferences, the needs of other users and the item attributes (S. S. Anand, B. Mobasher, 2005).Three parallel categories have emerged in the context of recommender systems: Collaborative filtering, content-based filtering and hybrid methods ( $\mathrm{J}$. Herlocker et all, 2004). Collaborative filtering is usually addressed under the assumption that if users rate items similarly or have similar tastes, they will rate other items similarly (M. Khaled, 2013; D. Jannach et all, 2011). Content-based filtering makes recommendations based solely on a user's profile built up by analyzing the content of items that the user has previously evaluated and/or user's profile and preferences (G. Adomavicius, A. Tuzhilin, 2005). Hybrid approaches combine aspects of both content-based and collaborative filtering (N. Belkin, W. Croft, 1992).

From those categories, collaborative filtering is still considered as the most promising and efficient technologies in practice (Y. Blanco-Fernandez et all, 2011; G. Karypis, 2001)Collaborative filtering solutions usually employ useritem rating matrix to make predictions and recommendations, avoiding by this way the need of providing extensive 
information about users and items. The prediction function estimates the preference of a user for an item that he/she has not experienced before. So depending on its value, we can then compute the approximate "top- $\mathrm{k}$ " recommendation lists.

A basic collaborative filtering scheme can be divided into three main parts:

\subsection{Neighborhood Formation}

When adopting collaborative filtering strategy, the crucial task is to identify users that are similar or neighbors of an active user. The neighbors refer to a set of like-minded users who share similar interests for items and its formation needs to employ the appropriate similarity metric. Pearson's correlation coefficient is one of the most frequently used measures in recommender systems to estimate the similarity between users (G. Karypis, 2001). Generally, the Pearson's correlation coefficient reflects the linear dependence between two variables and provides a value between -1 (i.e. low similarity) and 1 (i.e. high similarity) inclusive. The Pearson coefficient also considers user's individual rating behavior by incorporating the user's average rating. Moreover, there are other correlation coefficients such as cosine similarity and adjusted cosine similarity (G. Adomavicius, A. Tuzhilin, 2005; D. Jannach, 2011; G. Karypis, 2001). The pure cosine similarity measure computes the similarity between two rating vectors $\vec{u}$ and $\vec{v}$ and returns values between 0 (i.e. orthogonal vectors) and 1 (i.e. parallel vectors), where elevated values mean a high similarity. Unlike the Pearson coefficient, the pure cosine similarity does not take the average rating behavior of the users into account. One solution to overcome this weak point is to use the adjusted cosine similarity metric which factors out the user's average rating behavior and converts the similarity to a value between -1 and 1 inclusive, similar to the Pearson coefficient.

\subsection{Neighborhood Selection}

Now we come to the question of how to choose the neighborhood size $\mathrm{k}$ without compromising the prediction quality of our collaborative filtering algorithm (M. Khaled, 2013). If $\mathrm{k}$ is set up too large, we risk amplifying noise in the data since not all considered neighbors are relevant predictors. Additionally, we may see an increase in the time required for generating recommendations. On the contrary, a too small $\mathrm{k}$ can affect the prediction quality. A detailed discussion of the relative value of this tradeoff can be found in (S. S. Anand, B. Mobasher, 2005; M. Khaled, 2013)

\subsection{Aggregation of Ratings}

Lastly, the nearest neighbors' ratings for the target item are aggregated to form the final rating prediction value. The standard prediction formula has been proposed by (P.N. Tan, 2006).

\section{System Overview}

In today's world, significant organizational reforms occur in the field of advanced maritime transportation like in most other fields. The idea that has inspired our research turns out to be completely different from the ones reported in the literature.

\subsection{Context and Problem Description}

Despite all scientific and technological advances in the transportation system, many container terminals continue to suffer from practical mismanagements. And the transportation process is not necessarily resistant from this phenomenon which has multiple negative impacts on terminal performance.

Usually, disappointment with the organizational process is commonly expressed by straddles carriers' drivers, who often complain of favoritism being shown in assigning work schedules. This is essentially due to the very high demand for work schedule with small number of containers and small number of reshuffling movements in order to avoid investing too much physical effort and be less exposed to conflicts with customers.

The customers equally complain about the drivers who tend to come late to be assigned as reinforcement and avoid recording availability and checking customer identification when starting the work. Such practices may indeed cause severe disturbances during transportation process since there are no preventive measures in place to penalize "bad" drivers and reward "effective" ones. In such context, recommender systems have proved to be the best option to automatically identify appropriate work schedule assignments from a large number of alternatives, on the basis of some specified constraints and preferences.

In collaboration with the RADES container terminal, we identified 7 priority areas to focus when designing our recommender system:

- Transparency: Further increase transparency when assigning work schedules to drivers and guaranty the highest level of confidence among the transportation process.

- Punctuality: drivers are expected to arrive punctually for the shifts indicated in their allocation advice; this requires attendance 20 minutes prior to the transport start time.

- Performance monitoring: Provide an interactive system to judge driver's performance during transportation plans. Drivers must be aware that the rules governing conduct in transportation are to be strictly applied.

- Training: Provide guidance and direction of trainee drivers.

- Quick access: Minimize the length of the queue in the transport office.

- Less conflicts: Prevention and resolution of conflicts that can arise between drivers and customers (resp. staff).

- More comfort: Improve the comfort and the welfare of customers by providing the best possible conditions to undertake their containers (this includes ensuring that no disruption or distraction to customers comes from 
drivers themselves).

\subsection{Proposed Approach}

The recommender system we present in this paper is capable of intelligently assigning drivers to work schedules. A novel transportation management strategy will be discussed along with how the system is designed to support this. The general overview of the work schedule's recommendation system is given in Figure 1. As input, our system uses an e-exam database to load the driver's profiles (driver's ratings/scores, previously recorded conflicts) and all necessary details relative to work schedules and customers.

Given the drivers' previous ratings to some of their colleagues, similar drivers to the active driver are identified, and then, depending on the driver in charge, work schedules are recommended. This scenario will only happen if the total score associated to the active driver is greater than 10/20. Otherwise, he/she will be partnered with more experienced colleagues.

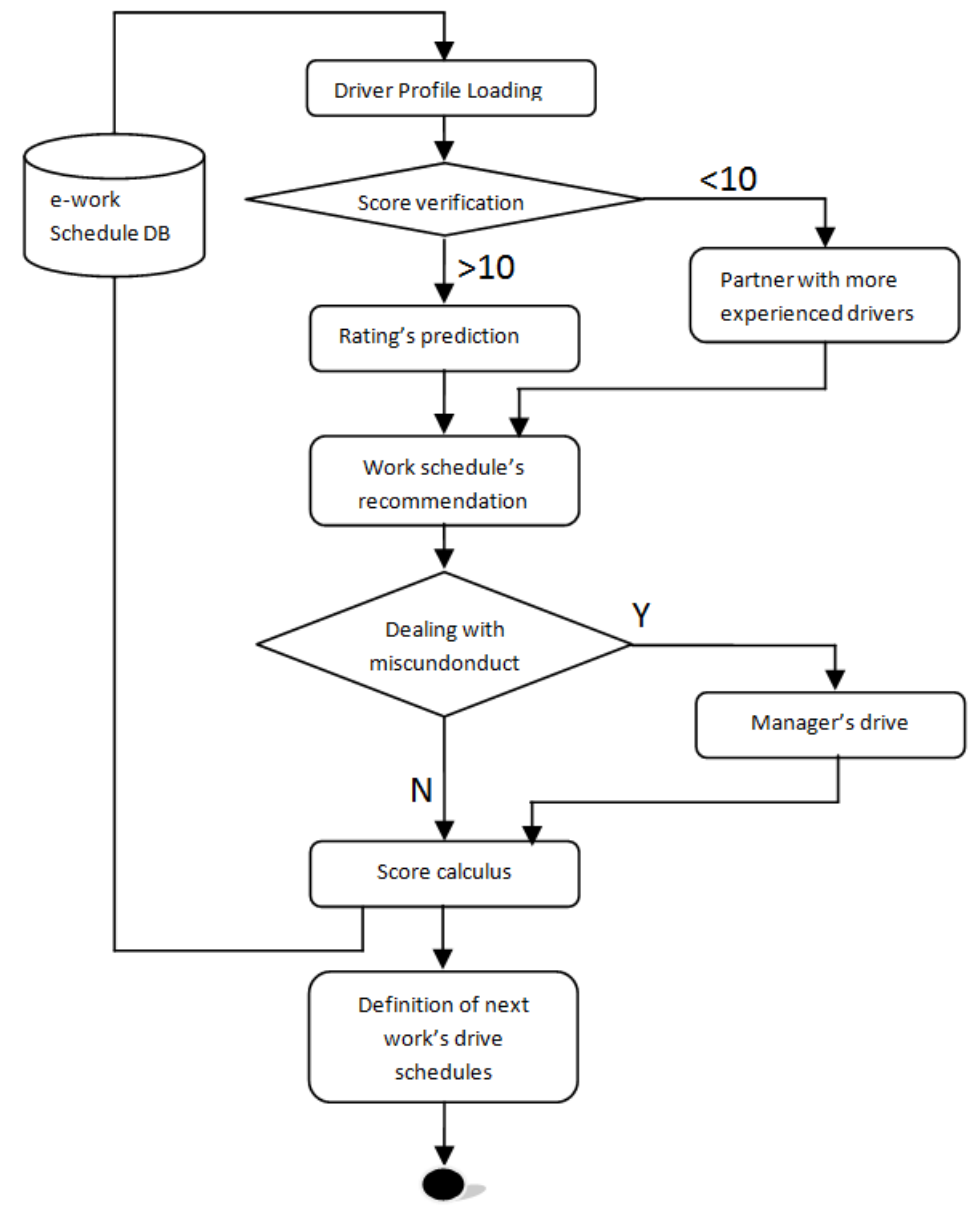

Figure 1. Work schedules' recommendation system flow chart.

This approach relies on neighbors' opinions, driver cumulative scores and eventual conflicts with customers. At the end of each transport plan, the drivers are asked to rate (in 1-5 rating scale) the work schedule suggested by the recommender system. More precisely, each driver's rate will be interpreted as a personal evaluation for the other drivers who are designated to accompany him/her during the transportation action plan. This is exactly the reason why the two dimensions of the rating matrix in table 1 involve only driver's identifiers.

Finally, for each driver, a total score will be computed based on various parameters (i.e. position, delays, absences, etc.). This score represents the most significant factor in the determination of the appropriate work schedules to suggest. Even, when all work schedules are provided, the system must be kept running until the end of the transportation action plan to ensure the recuperation of all manager's ratings. Our strategy follows an iterative scheme since it reprocesses those ratings to update the driver's scores, adapt the recommendations accordingly and define the future drive schedules.

Table 1. Driver-Driver rating matrix.

\begin{tabular}{lllllll}
\hline Driver ID & $\mathbf{1}$ & $\mathbf{2}$ & $\mathbf{3}$ & $\mathbf{4}$ & $\mathbf{5}$ & $\ldots$ \\
\hline 1 & & 4 & 5 & 1 & 4 & $\ldots$ \\
2 & 1 & & 3 & 4 & - & $\ldots$ \\
3 & - & 1 & & 3 & 1 & $\ldots$ \\
4 & 0 & 4 & 2 & & 1 & $\ldots$ \\
5 & 2 & - & 1 & 4 & & $\ldots$ \\
$\ldots$ & $\ldots$ & $\ldots$ & $\ldots$ & $\ldots$ & $\ldots$ & $\ldots$ \\
\hline
\end{tabular}


Table 2. Driver-Driver seniority-based similarity matrix.

\begin{tabular}{lllllll}
\hline Driver ID & $\mathbf{1}$ & $\mathbf{2}$ & $\mathbf{3}$ & $\mathbf{4}$ & $\mathbf{5}$ & $\ldots$ \\
\hline 1 & & -0.6 & 0.1 & 0.8 & -1 & $\ldots$ \\
2 & -0.6 & & 0.4 & -0.3 & 0.5 & $\ldots$ \\
3 & 0.1 & 0.4 & & -0.9 & 0.2 & $\ldots$ \\
4 & 0.8 & -0.3 & -0.9 & & 1 & $\ldots$ \\
5 & -1 & 0.5 & 0.2 & 1 & & $\ldots$ \\
$\ldots$ & $\ldots$ & $\ldots$ & $\ldots$ & $\ldots$ & $\ldots$ & $\ldots$ \\
\hline
\end{tabular}

\subsubsection{Problem Formulation \& Notations}

Our recommendation system uses a processing system configured to determine driver's scores using a collaborative rating process. Before proceeding further in describing the proposed approach, it is essential to have an understanding of how the transportation plan office often works in practice in order to extract relevant information and provide the necessary controls for ensuring the competency and effectiveness of the employees.

Once all drivers have joined their work schedules, the transport's managers start their inspection. The primary task for transport's managers, in addition to detecting any misconduct, is to ensure optimum transportation conditions in the work. Examples of driver's illegal behavior include making and receiving phone calls during the transportation plan or leaving the yard area.

Accordingly, when dealing with suspected misconduct, the transport's managers notify the director and the transport office in order to take the necessary measures. This policy appears to be effective, but is also vulnerable because of the informal way of establishing communication. Usually, drivers who are suspected of misconduct are never notified. This deprives them of a real opportunity to be corrected.

When designing our recommender system, we found some useful to use rating-based scores in order to formalize the future action plan in such situations. Specifically, the score calculation will take into account various data that can be explicitly provided by users (manager's ratings) or implicitly inferred from the drive's plan (position, delays, absences, etc.). For notational convenience, we give the definitions of the sets, parameters and the variables in Tables 3-5, respectively.

Table 3. The sets.

\begin{tabular}{ll}
\hline Sets & Definition \\
\hline$T$ & Set of scheduled transportation's sessions \\
$N(t)$ & Set of drivers for session t \\
$S(t)$ & Set of work schedules for session t \\
$E(t)$ & Set for not assigned work schedules for session t \\
$D(k)$ & Set of drivers assigned to the work schedule k \\
$R(t)$ & Set of the work schedules which need the assignment of \\
$O(v)$ & drivers \\
\hline
\end{tabular}

Table 4. The parameters.

\begin{tabular}{|c|c|}
\hline Parameters & Definition \\
\hline $\operatorname{Conf}(v, s)$ & $\begin{array}{l}1 \text { if a conflict is recorded betweendriver } \mathrm{v} \text { and the } \\
\text { customers who demand their containers in work } \\
\text { schedule s, } 0 \text { otherwise }\end{array}$ \\
\hline $\operatorname{Cap}(k)$ & The work schedule capacity \\
\hline
\end{tabular}

Table 5. The variables.

\begin{tabular}{|c|c|}
\hline Variables & Definition \\
\hline$r_{u, v}$ & The rating attributed by driver $\mathrm{u}$ to driver $\mathrm{v}$ \\
\hline$r_{i, v}^{*}$ & The rating attributed by the manager $\mathrm{i}$ to driver $\mathrm{v}$ \\
\hline$\hat{r}_{u, v}$ & $\begin{array}{l}\text { The rating attributed by driver } \mathrm{u} \text { to driver } \mathrm{v} \text { as predicted by } \\
\text { the model }\end{array}$ \\
\hline$\hat{R}_{u, k}$ & $\begin{array}{l}\text { The rating attributed by driver } \mathrm{u} \text { to the work schedule } \mathrm{k} \text { as } \\
\text { predicted by the model }\end{array}$ \\
\hline $\mathrm{Sen}_{v}$ & The driver's seniority \\
\hline$D e l_{v}^{t}$ & The recorded delay to join the work schedule in session $t$ \\
\hline $\operatorname{Pr} e s_{v}^{t}$ & The driver's presence state in session $t$ \\
\hline Score $_{v}^{t}$ & The driver's score in session $\mathrm{t}$ \\
\hline Score $_{v}$ & The accumulated driver's total score \\
\hline Work $_{v}^{t}$ & The work schedule assigned to driver $\mathrm{v}$ during session $\mathrm{t}$ \\
\hline
\end{tabular}

\subsubsection{Recommendation Engine}

Our collaborative filtering algorithm needs, as inputs, a first matrix representing the drivers' opinions on their colleagues (refer to table 1) and a second one for storing the seniority-based similarity between drivers (refer to table 2). Those similarities are calculated based on the attribute seniority when loading driver profiles and scattered into a [-1, 1] interval.

Table 6. Driver's scores interpretation.

\begin{tabular}{ll}
\hline Score & Interpretation \\
\hline $0 \leq$ Score $_{v}<5$ & $\begin{array}{l}\text { Recall the principle rules governing conduct in } \\
\text { work schedules }\end{array}$ \\
$5 \leq$ Score $_{v}<10$ & Partner with more experienced drivers \\
$10 \leq$ Score $_{v}<15$ & Varying the drive-drive assignments \\
$15 \leq$ Score $_{v}<20$ & No drive problem \\
\hline
\end{tabular}

Given an active driver, the objective of the resolution recommendation is to find the similar colleagues when there is no available rate for the drivers in the considered work schedule.

A straightforward approach is to apply the K-Nearest Neighbors strategy (P. Resnick et all, 2010) and select the set of "neighbors" of user $\mathrm{u}$, denoted by $\mathrm{N}_{\mathrm{u}}$, by choosing users who are sufficiently similar to u, i.e.

$$
\overrightarrow{N_{u}}=\{v \mid \operatorname{Sim}(u, v) \succ \gamma\}
$$

Where $\operatorname{Sim}(\mathrm{u}, \mathrm{v})$ is the similarity of $\mathrm{u}$ and $\mathrm{v}$ and is a threshold to select users who are qualified as "neighbors" of user $\mathrm{u}$. The neighbor selection is a very important step before prediction because the prediction ratings of an active user on items will be inaccurate if the selected neighbors are not sufficiently similar to the active user. Instead of selecting the top-k neighbors, we set a threshold for selecting neighbors. If the similarity of a candidate user $v$ and the active user $u$ is greater than or equal to the threshold, $\mathrm{v}$ will be selected into $\overrightarrow{N_{u}}$ (i.e. the set of "neighbors" of u).The rating similarities between drivers are determined based on the Pearson correlation coefficient. 


$$
\begin{aligned}
& \operatorname{Sim}_{R a t}(u, v)=\cos (u, v)=\frac{\vec{u} \cdot \vec{v}}{\|\vec{u}\| \cdot\|\vec{v}\|} \\
& =\frac{\sum_{i}\left(r_{u, i}-\overline{r_{u}}\right) \times\left(r_{v, i}-\overline{r_{v}}\right)}{\sqrt{\sum_{i}\left(r_{u, i}-\overline{r_{u}}\right)^{2}} \times \sqrt{\sum_{i}\left(r_{v, i}-\overline{r_{v}}\right)^{2}}}
\end{aligned}
$$

And the total similarity will be computed as follows:

$$
\operatorname{Sim}(u, v)=\frac{\alpha \cdot \operatorname{Sim}_{R a t}(u, v)+\beta \cdot \operatorname{Sim}_{S e n}(u, v)}{2}
$$

Where $\alpha$ and $\beta$ are inversely proportional constants.

The prediction of an unknown rating $\hat{r}_{u, i}$ requires the search of users $v$ that are similar to $u$, or the nearest neighbors of $u$ according to cosine similarity measure

$$
\hat{r}_{u, i}=\bar{r}_{i}+\frac{\sum_{j \in N(i)} \operatorname{Sim}(i, j) \times\left(r_{u, j}-\bar{r}_{j}\right)}{\sum_{j \in N(i)} \operatorname{Sim}(i, j)}
$$

The final rating of user $u$ to the work schedule $k$ is given by:

$$
\hat{R}_{u, k}=\sum_{u \in D(k)} \hat{r}_{u, v}
$$

Finally, the score computed for each driver will be

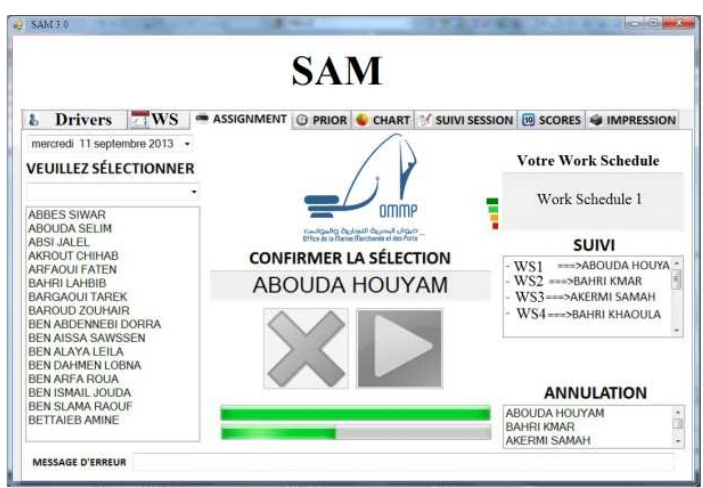

A

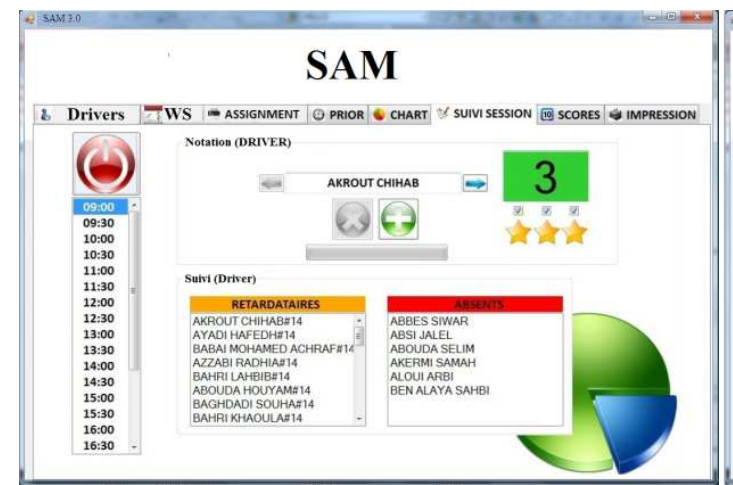

$\mathrm{C}$ expressed as follows:

$$
\operatorname{Score}_{u}^{t}= \begin{cases}\frac{r_{u}^{* t}+\operatorname{Sen}_{u}^{t}}{1-\operatorname{Del}_{u}^{t}} & \text { if } \operatorname{Pr} e s_{u}^{t}=1 \\ 0 & \text { otherwise }\end{cases}
$$

Where $t \in T, u \in N_{t}$.

The calculated scores can be then interpreted as shown in table 6 and reused when defining the future work schedule's driver. So the more the total score is low, the more driven plans will be attributed to the driver.

A more formal description of the proposed approach can be found in Algorithm 1.

\section{Implementation of the Proposed Approach}

The recommender system, which we named the Straddle carrier Assignment Model (SAM) software, is already finished and it has been successfully tested by the RADES transport's office. The preliminary results show a good performance in terms of recorded conflicts and drivers' (resp. transportation's staff) satisfaction. The success of our solution has also inspired other Tunisian container terminals to adopt and replicate its model for their own organizational needs.

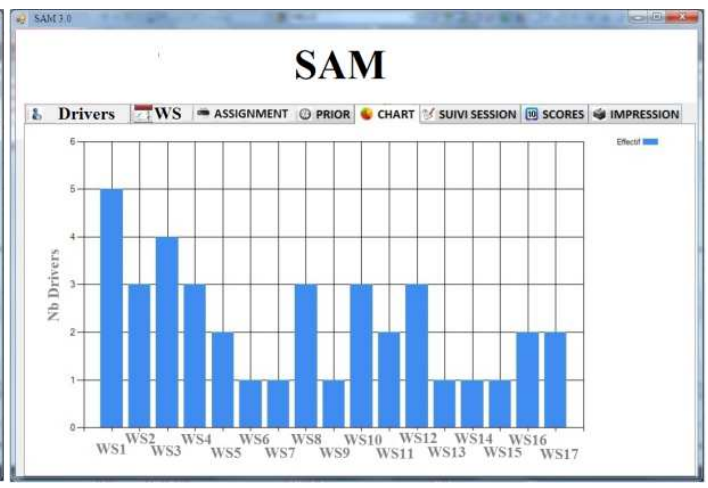

B

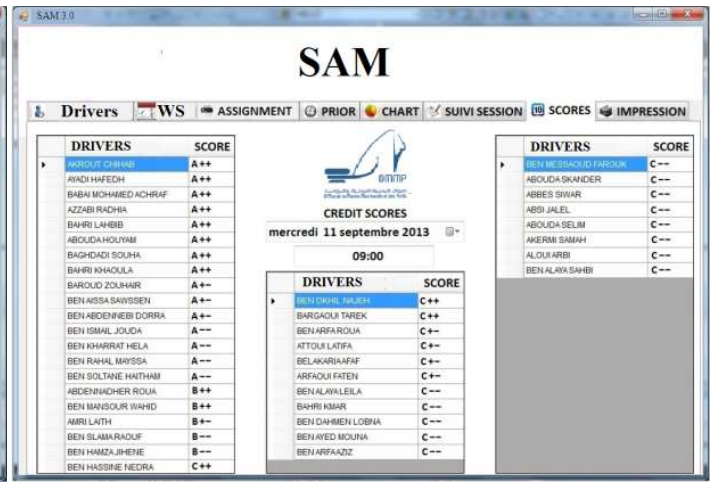

Figure 2. Sample screens from the SAM software.

To design our recommender system, modular three-tier architecture (client, server and back-end database) is 
employed. On the client side, SAM provides the users (transportation's office agents / managers and RADES director) with multi-task interfaces.

On the server side, intelligent module performs the calculation of all necessary scores and utilities. The back-end e-work schedule database stores preferences and information about more than 250 straddle carriers' drivers.

The system was implemented in a Microsoft Windows environment using Microsoft Visual Studio.Net for the development of our front-end system. The back-end database was managed using MySQL. The software implements the following main functions:

- Suggestive module: The interactive interface in Figure 2.A is projected on the wall providing visual feedback to all drivers who joined the queue in the transportation's office. The recommendation process must be undertaken with total transparency.

- Graphical tools: To facilitate verification, interpretation and validation of the system recommendations, graphical display tools (See Figure 2.B) are provided to the users. Although the procedure is totally automatic in normal use, the user can manually intervene in urgent circumstances. In such situations, the graphical representations can be of great use.

- Rating system: At the end of each transporter's plan, managers and supervisors return to the system to enter their observations in the form of ratings (See Figure 2.C).

- Score's generator: When all data are collected, the system generates the list of the updated driver's scores as shown in Figure 2.D.

The development and implementation of the proposed expert system is in direct congruence with several of the strategic goals of the RADES container terminal. The SAM software created a competitive advantage, increased job satisfaction and greatly reduced conflicts. Customers are also more satisfied because the system is in place. Time savings are significant, recorded delays when starting work schedules became quasi nonexistent and a social equilibrium is expected to take place in the short-term future.

\section{Results}

To evaluate the SAM project, we conduct a series of faceto-face interviews with the administration staff and posted a questionnaire on the RADES web site forum page. The results collected from the analysis concern all drive positions (replacement drivers, part-time drivers, contractual drivers...). According to the RADES transport's regulations, some volunteers can be engaged as managers.

We received a total of 24 valid responses. The questionnaire focused on the drivers' perceptions of how the SAM software influenced the conduct of transportations and were specifically designed to obtain feedbacks on four aspects: reasonableness, accuracy, equity and transparency of the work schedule's assignments.

The obtained results in Figure 2 revealed the paradoxical effects of applying our recommender system. The high experienced drivers are often favored ahead of their colleagues. The favoritism comes in the form of a monopoly of simple work schedules. Such situation gave rise to conflicts with the other drive profiles and indeed also to tensions between all the transportation's staff.

With its adoption in the year 2012, the SAM has completely changed the rules by treating all drivers the same without any discrimination. But we should realize that our system had not been welcomed by some drivers who considered it as an infringement of an acquired right and many of them have chosen to boycott it in response. Despite the remarkable decrease in the total number of recorded conflicts (about $30 \%$ ) in the year 2013, the mean scores obtained from the questionnaires in Figure 4 still reveal a significant difference in the responses of the two groups. Unfortunately, the SAM project is still struggling to persuade users to change their behaviors but we expect that this situation will not persist for long especially since we have introduced the scientific methodology that we have adopted for the construction of our models.

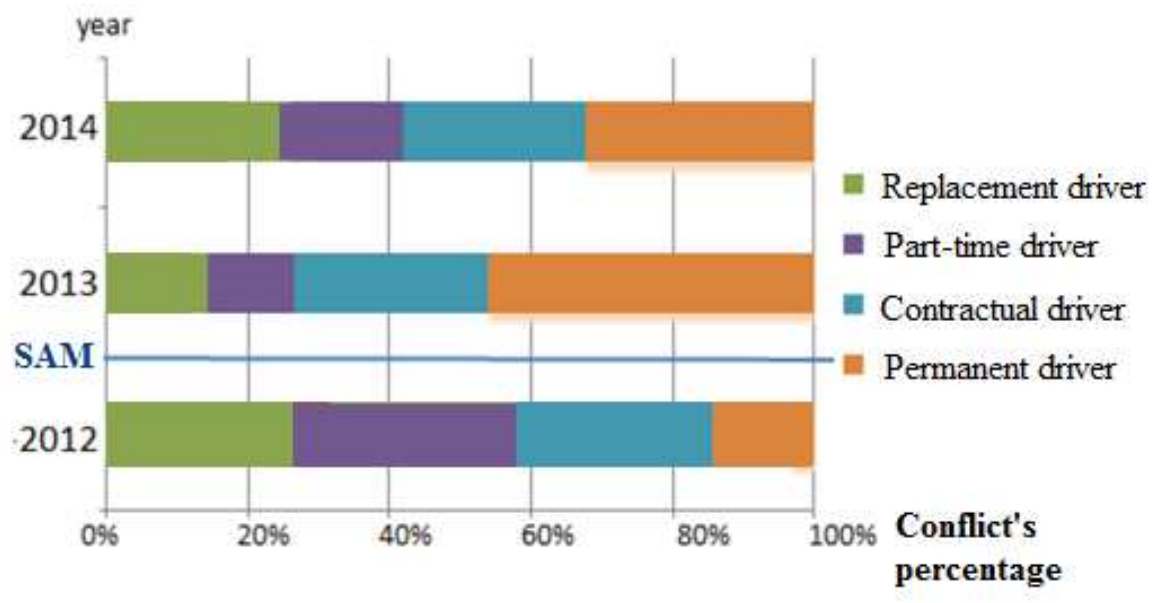

Figure 3. Percentages of registered conflicts during work schedule assignments. 


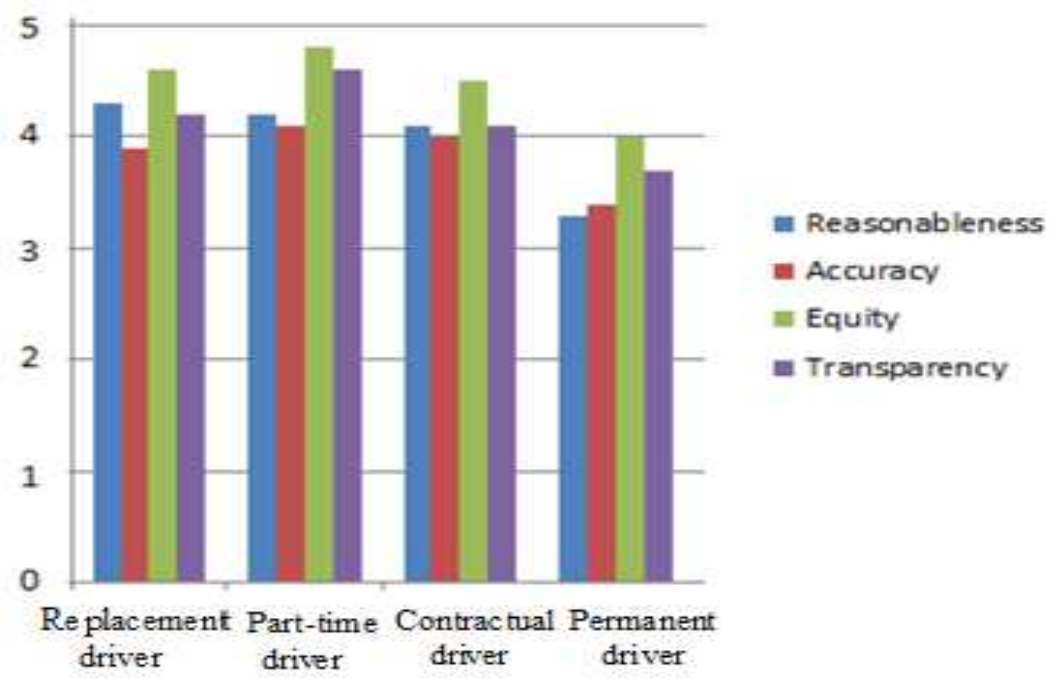

Figure 4. Qualitative evaluations of the SAM recommendations.

\section{Conclusion}

In this paper, we presented our design and implementation of a recommender system that can help straddle carrier's drivers select appropriate work schedule based on their preferences, scores and transport constraints. The proposed recommendation method is further strengthened by reusing all relevantly similar previous experiences to make the current suggestion more accurate. The SAM system is an initial attempt towards a more complete software package for automatically managing transportation of containers in container terminals.

Algorithm1 SAM collaborative filtering algorithm

Require: Driver-driver rating matrix, Driver-driver seniority-based similarity matrix, driver's profile information. repeat

Random selection of a work schedule $k \in E(t)$

If Conflict (Driver $u$, Customer $s \in k=\varnothing$ then

Assign $u$ to $k$

end if

until $E(t)=\varnothing$

foreach driver $u$ do

Load the driver $u$ 's profile

if Score $_{u} \succ 10$ then

for each work schedule $k$ do

for each driver $w$ in work schedule $k$ do

Find the nearest drivers to $u$ who have rated driver $w$

Predict the rating $\hat{r}_{u, w}$ for driver $u$ on driver $w$

end for

Predict the rating $\hat{R}_{u, k}$ for driver $u$ on work schedule $k$

if Conflict (Driver $u$, Customer $s \in k=\varnothing$ then

Recommend work schedule with maximum $\hat{R}_{u, k}$

end if

end for

else

Partner with more experienced colleagues end if

end for

\section{References}

[1] G. Adomavicius , A. Tuzhilin."Toward the next generation of recommender systems: a survey of the state-of-the-art and possible extensions", IEEE Computer Society, volume 17, Issue 6. (2005).

[2] S. S. Anand, B. Mobasher., "Lecture Notes in Computer Science", Springer Berlin Heidelberg, volume 3169: 1$36,(2005)$

[3] N. Belkin, W. Croft. "Information filtering and information retrieval: Two sides of the same coin?" Communications of the ACM, 35(12):29-38, (1992).

[4] Y. Blanco-Fernandez, M. L. Nores, J. J. P. Arias, and J. G. Duque. "An improvement for semantics-based recommender systems grounded on attaching temporal information to ontologies and user profiles". Eng. Appl. of AI, 24(8):13851397, (2011).

[5] J. Herlocker, J. Konstan, L. Terveen, and J. Riedl. "Evaluating collaborative filtering recommender systems". ACM Transactions on Information Systems, 22(1):5-53, (2004).

[6] D. Jannach, M. Zanker, A. Felfernig, and G. Friedrich. "Recommender Systems: An Introduction". Cambridge University Press, (2011).

[7] G. Karypis. "Evaluation of item-based top-n recommendation algorithms". In Proceedings of the tenth international conference on Information and knowledge management, pages 247-254, Atlanta, Georgia, USA, ACM Press, (2001).

[8] M. Khaled, "Six Sigma Approach for the Straddle Carrier Routing Problem", Journal of Maritime Research, vol. X. No.1 (2013), pp. 71-84, (2013).

[9] M. Khaled, T. Sadraoui "Optimizing the Operational Process at Container Terminal" International Journal of Econometrics and Financial Management, Vol. 3, No. 2, 91-98, (2015). 
[10] M. Khaled, M. Faissal, "Genetic procedure for the Single Straddle Carrier Routing Problem" International Journal of Advanced Computer Science and Applications, Vol. 3, No. 11, (2012).

[11] P. Melville and V. Sindhwani. "Recommender Systems". Encyclopedia of Machine Learning, pages 829-838, (2010).
[12] P. Resnick, N. Iacovou, M. Suchak, P. Bergstrom, and J. Riedl. Grouplens, "An open architecture for collaborative filtering of net news", Proceedings of ACM 1994 Conference on Computer Supported Cooperative Work, pages 175-186. ACM Press, (1994).

[13] P.-N. Tan, M. Steinbach, and V. Kumar. "Introduction to Data Mining". Addison Wesley, (2006). 